\title{
VEJ $\phi$, a novel filamentous phage of Vibrio cholerae able to transduce the cholera toxin genes
}

\author{
Correspondence \\ Javier Campos \\ javier.campos@cnic.edu.cu
}

Received 29 June 2009

Revised 10 September 2009

Accepted 14 October 2009

\author{
Javier Campos, Eriel Martínez,† Yovanny Izquierdoł and Rafael Fando
}

Department of Molecular Biology, National Center for Scientific Research, Ave 25 y 158 no. 15202, Cubanacán, Playa, Ciudad de la Habana 12100, PO Box 6990, Cuba

\begin{abstract}
A novel filamentous bacteriophage, designated $\mathrm{VEJ} \phi$, was isolated from strain MO45 of Vibrio cholerae of the $\mathrm{O} 139$ serogroup. A molecular characterization of the phage was carried out, which included sequencing of its whole genome, study of the genomic structure, identification of the phage receptor, and determination of the function of some of the genes, such as those encoding the major capsid protein and the single-stranded DNA-binding protein. The genome nucleotide sequence of VEJ $\phi$, which consists of $6842 \mathrm{bp}$, revealed that it is organized in modules of functionally related genes in an array that is characteristic of the genus Inovirus (filamentous phages). VEJ $\phi$ is closely related to other previously described filamentous phages of $V$. cholerae, including VGJ $\phi$, VSK and fs1. Like these phages, VEJ $\phi$ uses as a cellular receptor the type IV fimbria called the mannose-sensitive haemagglutinin (MSHA). It was also demonstrated that $\operatorname{VEJ} \phi$, like phage VGJ $\phi$, is able to transmit the genome of phage CTX $\phi$, and therefore the genes encoding the cholera toxin (CT), horizontally among populations of $V$. cholerae expressing the MSHA receptor fimbria. This suggests that the variety of phages implicated in the horizontal transmission of the CT genes could be more diverse than formerly thought.
\end{abstract}

\section{INTRODUCTION}

Filamentous bacteriophages belong to the genus Inovirus, members of which infect almost exclusively Gram-negative bacteria. The virions of these phages are slender filaments usually about $6 \mathrm{~nm}$ in diameter and 800-2000 nm long, comprising a helical capsid of several thousand copies of the major coat protein (pVIII) surrounding a single-stranded circular DNA genome about of 5-8 kb, encoding around 10 genes (Marvin, 1998). These phages usually use type IV pili as receptors to infect bacterial cells (Marvin, 1998).

Vibrio cholerae, the Gram-negative bacterium causing the acute diarrhoeal disease cholera, hosts several filamentous phages (Campos et al., 2003b; Ehara et al., 1997; Faruque et al., 2005; Jouravleva et al., 1998; Kar et al., 1996; Waldor \& Mekalanos, 1996). Some of them are able to transduce portions of alien DNA among bacterial populations, including the transmission of virulence genes mediated by different mechanisms of horizontal gene transfer (Campos et al., 2003a; Davis \& Waldor, 2003; Faruque

Abbreviations: CT, cholera toxin; MSHA, mannose-sensitive haemagglutinin; RF, replicative form; TCP, toxin co-regulated pilus.

tPresent address: Universitat de Barcelona, Av. Diagonal 645, 08028 Barcelona, Spain.

‡Present address: Instituto de Biotecnología de las Plantas, Universidad Central 'Marta Abreu' de Las Villas, Santa Clara 54830, Villa Clara, Cuba.

The GenBank/EMBL/DDBJ accession number for the nucleotide sequence of VEJ $\phi$ is FJ904927. et al., 2002). In this respect, most significant was the finding that the genes encoding cholera toxin $(\mathrm{CT})$, the main virulence attribute of $V$. cholerae, are contained in the genome of the CTX $\phi$ phage, which is integrated in the chromosome of toxigenic strains of this bacterium at the chromosomal dif site (Huber \& Waldor, 2002; Val et al., 2005; Waldor \& Mekalanos, 1996). CTX $\phi$ is also able to replicate directly from the bacterial chromosome and to produce infective phage particles with potential for transducing the CT genes to non-pathogenic environmental strains (Davis \& Waldor, 2003; Waldor \& Mekalanos, 1996). The receptor of this phage is the toxin co-regulated pilus (TCP), a type IV fimbria essential for colonization of the human intestine by $V$. cholerae (Waldor \& Mekalanos, 1996). The genes required for TCP expression and assembly at the bacterial surface are located in the vibrio pathogenicity island (VPI), which has also been horizontally acquired by this pathogen during evolution, although its mechanism of transmission is still controversial (Faruque et al., 2003; Karaolis et al., 1998; Karaolis \& Kaper, 1999; Lee, 1999; Rajanna et al., 2003).

More recently, another filamentous phage was described VGJ $\phi$ - which is also able to transmit the CT genes by means of a mechanism of specialized transduction, and it uses the type IV pilus mannose-sensitive haemagglutinin (MSHA) as a receptor (Campos et al., 2003a, b). The genomes of VGJ $\phi$ and CTX $\phi$ are able to recombine when both phages co-infect the same cell. As a result, a hybrid genome is formed that is packaged into the sheath of VGJ $\phi$, 
forming a hybrid phage particle designated $\mathrm{HybP} \phi$, which is able to infect other vibrio cells via the MSHA receptor (Campos et al., 2003a). Thus, CTX $\phi$ and therefore the CT genes it carries are transduced by VGJ $\phi$ through a mechanism independent of the TCP receptor (Campos et al., 2003a). Hybrid phage HypP $\phi$ could potentially infect vibrios that do not express TCP; once inside the cells phage HypP $\phi$ is capable of integrating into the bacterial chromosome, guaranteeing the vertical perpetuation of the acquired CT genes (Campos et al., 2003a).

Studies on the horizontal gene transfer mechanisms of CT genes and other virulence-related genes have been of great importance in understanding the evolution of the virulent strains of $V$. cholerae. This knowledge has allowed researchers to gain new perspectives on the possible epidemiological trends that cholera disease could follow in the future and it has had a direct influence on the strategies currently applied in the design of new vaccines based on live attenuated strains of $V$. cholerae, in the sense of trying to avoid the reacquisition of virulence genes by these strains in the environment (Campos et al., 2003a; Kimsey \& Waldor, 1998).

In this study we characterize phage VEJ $\phi$, a new filamentous phage hosted by $V$. cholerae that is a close relative of VGJ $\phi$, and we explore its ability to transduce the CT genes among bacterial populations of this pathogen.

\section{METHODS}

Strains, plasmids, phages and culture media. Strains, plasmids and phages used in this study are described in Table 1. Bacterial strains were grown in Luria-Bertani medium (LB). It was normally adjusted to $\mathrm{pH} 7.0$ or to $\mathrm{pH} 6.5$ for expression of the TCP in classical strains of $V$. cholerae. Ampicillin $\left(100 \mu \mathrm{g} \mathrm{ml}{ }^{-1}\right)$ or kanamycin $(50 \mu \mathrm{g}$ $\mathrm{ml}^{-1}$ ) were added when required.

Isolation of phage VEJ $\boldsymbol{\phi}$. Strain MO45 was grown in $5 \mathrm{ml} \mathrm{LB}$ in a $25 \mathrm{ml}$ Erlenmeyer flask for $6 \mathrm{~h}$ with shaking (240 r.p.m.) at $37^{\circ} \mathrm{C}$. One millilitre of this culture was serially filtered through $0.45 \mu \mathrm{m}$ and $0.22 \mu \mathrm{m}$ pore-size filters (Sartorius). An aliquot $(50 \mu \mathrm{l})$ of the filtered

Table 1. Strains, plasmids and phages used in this study

\begin{tabular}{|c|c|c|}
\hline $\begin{array}{l}\text { Strain, plasmid, } \\
\text { phage or primer }\end{array}$ & Description or sequence & Origin or reference \\
\hline E. coli & & Promega \\
\hline JM109 & recA1 supE44 endA1 hsdR17 gyrA96 relA1 thi $\Delta($ lac proAB) & \\
\hline \multicolumn{3}{|l|}{ V. cholerae } \\
\hline $569 \mathrm{~B}$ & O1, Classical, Inaba & Calcutta, India, 1945 \\
\hline C6706 & O1, El Tor, Inaba & Peru, 1991 \\
\hline KHT46 & C6706 $\Delta m s h A 1$ & Thelin \& Taylor (1996) \\
\hline KHT52 & $\mathrm{C} 6706 \Delta t c p A 10$ & Thelin \& Taylor (1996) \\
\hline MO45 & O139 & Madras, India, 1993 \\
\hline \multicolumn{3}{|l|}{ Plasmids } \\
\hline pIJ2921 & General-purpose vector & Mendez \& Chater (1987) \\
\hline $\mathrm{p} 4$ & $\begin{array}{l}\text { pIJ2921 derivative containing a } 435 \text { bp HincII fragment from the RF of VEJ } \phi \text {, inserted at } \\
\text { SmaI site of vector }\end{array}$ & This study \\
\hline p5 & $\begin{array}{l}\text { pIJ2921 derivative containing a } 1039 \text { bp HincII fragment from the RF of VEJ } \phi \text {, inserted at } \\
\text { SmaI site of vector }\end{array}$ & This study \\
\hline p11 & $\begin{array}{l}\text { pIJ2921 derivative containing a } 1787 \text { bp HincII fragment from the RF of VEJ } \phi \text {, inserted at } \\
\text { SmaI site of vector }\end{array}$ & This study \\
\hline \multicolumn{3}{|l|}{ Phages } \\
\hline CTX-Kn $\phi$ & CTX $\phi$ containing a $\mathrm{Kn}^{\mathrm{r}}$ gene inserted into the unique NotI site of the phage genome & Campos et al. (2003a) \\
\hline VEJ $\phi$ & Filamentous phage of $V$. cholerae & This study \\
\hline \multicolumn{3}{|l|}{ Primers } \\
\hline EJ1 & 5'-GGAAGAAGCAACGATTGTCG-3' & This study \\
\hline EJ2 & 5'-CCGTATTGAGCAAACTTCTC-3' & This study \\
\hline EJ3 & 5'-CTATGAGCAAGAAAGCGAGG-3' & This study \\
\hline EJ4 & 5'-CTCGACTCAATTCTGACAAC-3' & This study \\
\hline EJ5 & 5'-CATTTCGCTTCGATTCATTG-3' & This study \\
\hline EJ6 & 5'-ACTCATAGAACCAATGGATG-3' & This study \\
\hline EJ7 & 5'-GCTTAGTTCGTCTCGACTTG-3' & This study \\
\hline EJ8 & 5'-GTAATGGCTAACATCACTGG-3' & This study \\
\hline EJ9 & 5'-CATCGGTGATACCAGAGAAG-3' & This study \\
\hline EJ10 & 5'-GTGATCATCAATACTCTTGC-3' & This study \\
\hline NJ-4 & 5'-CCTGTAGAAATTCCGTCTCC-3' & Campos et al. (2003a) \\
\hline CTB1 & 5'-GCGATTGAAAGGATGAAGG-3' & Campos et al. (2003a) \\
\hline
\end{tabular}


supernatant was plated on solid LB to check for sterility. Another aliquot $(20 \mu \mathrm{l}$ ) was mixed with $20 \mu \mathrm{l}$ of a culture of 569B (grown under the same conditions as MO45) and incubated for $20 \mathrm{~min}$ at room temperature. The mixture was then inoculated into $100 \mathrm{ml} \mathrm{LB}$ in a $500 \mathrm{ml}$ Erlenmeyer flask and grown at $37{ }^{\circ} \mathrm{C}$ with shaking (240 r.p.m.) overnight. The culture was centrifuged (8000 g, $20 \mathrm{~min}$ ), and the supernatant was filtered through a $0.45 \mu \mathrm{m}$ pore-size filter. Phage particles in the filtrate were precipitated by addition of $\mathrm{NaCl}$ and polyethylene glycol to final concentrations of 3 and $5 \%(\mathrm{w} / \mathrm{v})$, respectively. The mixture was incubated on ice for $30 \mathrm{~min}$ and centrifuged at $12000 \mathrm{~g}$ for $20 \mathrm{~min}$. The supernatant was discarded, and the phage-containing pellet was resuspended in $1 \mathrm{ml}$ PBS.

DNA isolation and manipulation. Plasmid DNA was prepared by using the Wizard plus SV system (Promega), and genomic ssDNA from phage particles was prepared as described by Sambrook et al. (1989). DNA restriction and modification enzymes were used according to the manufacturer's instructions (Promega). The DNA was electrophoresed on $0.8 \%(\mathrm{w} / \mathrm{v})$ agarose gels and was visualized with ethidium bromide $\left.(1 \mu \mathrm{g} \mathrm{ml})^{-1}\right)$ or with acridine orange $(1 \mu \mathrm{g}$ $\mathrm{ml}^{-1}$ ), which fluoresces green with double-stranded DNA and orange with ssDNA (McMaster \& Carmichael, 1977). Sequencing reactions were ordered from Macrogen. The whole sequence of the VEJ $\phi$ genome was assembled by combining data obtained from sequencing of cloned fragments of the replicative form (RF) in the pIJ2921 vector with modified universal and reverse primers and from direct sequencing of the purified genomic ssDNA of the phage (plus strand) or the RF with primers EJ1-EJ10 (Table 1).

Nucleotide and protein sequence analysis and alignment. Sequences were assembled and analysed by using Vector NTI advance 10 (Invitrogen), which was also used for alignment analyses. The BLAST program was used to search for homologous DNA or protein sequences in databases.

Electron microscopy. Purified phage particles were negatively stained with $4 \%(\mathrm{w} / \mathrm{v})$ uranyl acetate and mounted on freshly prepared Formvar grids, which were examined with a JEOL JEM2000EX transmission electron microscope.

Infection assay. VEJ $\phi$ or CTX-Kn $\phi$ donor strains were grown in LB to an $\mathrm{OD}_{600}$ of 1 measured in a BioPhotometer (Eppendorf). One millilitre of culture was filtered through a $0.22 \mu \mathrm{m}$ pore-size filter (Sartorius). Aliquots $(100 \mu \mathrm{l})$ of pure or serially diluted cell-free supernatant of the donor were mixed with $20 \mu \mathrm{l}\left(\sim 10^{8}\right.$ cells $)$ of a culture of the recipient strain grown in $\mathrm{LB}$ for $6 \mathrm{~h}$ at $37^{\circ} \mathrm{C}$ and 240 r.p.m. The mixture was incubated for $20 \mathrm{~min}$ to allow infection and then plated onto a soft LB agar $(0.4 \%)$ overlay in the case of VEJ $\phi$ or onto solid LB plates supplemented with kanamycin (LBKn) in the case of CTX-Knф. The number of opaque plaques or $\mathrm{Kn}^{\mathrm{r}}$ c.f.u. was determined at $20 \mathrm{~h}$. Alternatively, the infection mixture was seeded directly into LB broth if necessary.

Protein methods. Preparations of phage proteins were analysed by SDS-PAGE. For amino-terminal sequencing, the proteins were first separated by SDS-PAGE, then electro-transferred to a PVDF membrane. After transfer the membrane was stained with Coomassie blue $(0.1 \%$ in $50 \%$ methanol) for $5 \mathrm{~min}$ and subsequently destained with $50 \%$ methanol until the protein bands were clearly visible (5-10 $\mathrm{min})$. The membrane was washed twice for $5 \mathrm{~min}$ with distilled water and the bands of interest were cut from the membrane with a new scalpel. The piece of membrane with each band was used for amino-terminal protein sequencing by the Edman degradation method using an Applied Biosystems Procise automatic sequencer.

Nucleotide sequence accession numbers. The nucleotide sequence of VEJ $\phi$ has been deposited in the GenBank database under accession number FJ904927.

\section{RESULTS AND DISCUSSION}

\section{Isolation of the new filamentous phage VEJ $\phi$}

We noticed the presence of an extrachromosomal genetic element in total DNA preparations of $V$. cholerae O139 strain MO45. We investigated if this episome corresponded to the genome of a phage. Cell-free culture supernatants of MO45 were used to infect the phage-free indicator strain 569B, which acquired the same episome, as checked by restriction analysis (data not shown). The transmission of the episome from MO45 to 569B without direct cell-cell contact indicated the presence of a phage in the supernatants of MO45. Restriction analyses showed that this episome was a circular, double-stranded DNA molecule with an approximate size of $7 \mathrm{~kb}$ (data not shown). The restriction map generated was different from those previously described for $V$. cholerae phages, so we named this new phage VEJ $\phi$.

VEJ $\phi$ phage particles were purified from culture supernatants of infected 569B and their genetic material was isolated. It was resistant to hydrolysis with several restriction enzymes and RNase, but was susceptible to mung-bean nuclease degradation (data not shown), and it fluoresced orange when it was electrophoresed in the presence of acridine orange (data not shown), indicating that the nucleic acid extracted from phage particles was ssDNA.

Phage suspensions of VEJ $\phi$ were able to produce opaque plaques when spread onto a lawn of the sensitive strain 569B grown on soft agar, which allowed the titration of the phage. The mean titre from three independent experiments was $7.4 \times 10^{10}$ p.f.u. $(\mathrm{ml} \text { culture } \times \mathrm{OD} \text { unit })^{-1}$, indicating that it was a very prolific phage, comparable in this respect to VGJ $\phi$ and M13. The ability of VEJ $\phi$ to form opaque plaques, taken together with the size and the singlestranded nature of its genome, suggested this was a filamentous phage. This hypothesis was confirmed by visualization of the phage particles by electron microscopy (not shown), as well as by the DNA sequence similarity of the VEJ $\phi$ genome with those of other filamentous phages stored in DNA databases.

\section{Genomic organization}

The VEJ $\phi$ DNA sequence is similar to sequences of other previously described phages, such as VSK (93.1\%), fs1 $(89.9 \%)$ and VGJ $\phi(84.2 \%)$. The whole genome of VEJ $\phi$ consisted of 6842 nucleotides and had a G $+\mathrm{C}$ content of $42.95 \mathrm{~mol} \%$, which is slightly lower than the $\mathrm{G}+\mathrm{C}$ content of its $V$. cholerae host $(47.7 \%$ for chromosome I and $46.9 \%$ for chromosome II). The sequence of VEJ $\phi$ deposited in the GenBank database (FJ904927) corresponds to the positive DNA strand of the phage, i.e. the same strand that is packaged in the phage capsid. This was confirmed because only oligonucleotides hybridizing with this DNA strand exhibited sequence reactions when phage genomic ssDNA was used as the template. 
The VEJ $\phi$ genome has 11 putative ORFs organized in four modular structures: the replication module, the structural module, the assembly module and the regulation module (Fig. 1). The replication module contains ORF361 and ORF112 (Fig. 1). ORF361 is similar to rolling circle replication proteins of other filamentous phages (VGJ $\phi$, $95.8 \%$; KSF, 76.2\%; CTX $\phi, 34.1 \%$ similarity), while ORF112 is identical to the same ORF of VGJ $\phi$ and is similar in size and position to ssDNA-binding proteins of other inoviruses. Indeed we demonstrated that the protein product of ORF112 functions as the ssDNA-binding protein of VEJ $\phi$ and VGJ $\phi$, by retardation assays, which showed that it binds specifically to the phage genomic ssDNA (data not shown; while this paper was under revision, the purification and characterization of the ORF112 product was published by Falero et al., 2009). Thus, we propose to rename the ORF112 gene of VEJ $\phi$ and $\mathrm{VGJ} \phi$ as $\mathrm{gV}^{\mathrm{VEJ} \phi}$ and $\mathrm{gV}^{\mathrm{VGJ} \phi}$ and their respective proteins as $\mathrm{pV}^{\mathrm{VEJ} \phi}$ and $\mathrm{pV}^{\mathrm{VGJ} \phi}$, to follow the denomination of canonical phages of the Inovirus genus as the fd group.

The structural module comprises ORF81, ORF44, ORF29, ORF493 and ORF104 (Fig. 1), whose protein products are similar in size, genomic position and/or sequence similarity to putative or proved capsid proteins of other inoviruses, suggesting that their protein products should accomplish the same function in VEJ $\phi$. A supporting example is ORF44, whose product was identified as the major capsid protein of VEJ $\phi$ and VGJ $\phi$ in this work by amino-terminal sequencing of these peptides. The predicted amino acid sequence of ORF44 from VEJ $\phi$ differs in only one residue at position 23 from the same ORF of VGJ $\phi$, where a serine residue was substituted by an alanine. Whole particles of VEJ $\phi$ and VGJ $\phi$ were electrophoresed in SDS-PAGE (Fig. 2) and the major capsid proteins were used for sequencing by Edman degradation as described in Methods. The first ten amino acid residues of ORF44 (MADIFGAIDF) from both phages were identified. Consequently, we propose to rename the ORF44 genes of VEJ $\phi$ and VGJ $\phi$ as gVIII ${ }^{\mathrm{VEJ} \phi}$ and gVIII ${ }^{\mathrm{VGJ} \phi}$, and the major capsid proteins as pVIII ${ }^{\mathrm{VEJ} \phi}$ and $\mathrm{pVIII}{ }^{\mathrm{VGJ} \phi}$, to follow the denomination of canonical phages of the Inovirus genus.

As we have predicted in previous work (Campos et al., 2003b), no leader peptide is present in the ORF44 product. Other filamentous phages such as Pf3 and C2 have major capsid proteins without leader peptides (Kiefer et al., 1997; Kostrikis et al., 1995), which are inserted into the bacterial inner membrane independent of the Sec translocase machinery, driven by hydrophobic forces assisted by YidC protein (Kiefer \& Kuhn, 1999; Serek et al., 2004). Since the ORF44 product lacks a leader peptide, we predict that a similar mechanism mediates the insertion of this protein into the inner membrane of $V$. cholerae.

The assembly module of VEJ $\phi$ only contains ORF411 (Fig. 1 ), whose protein product is similar to the assembly protein of other filamentous phages such as VGJ $\phi$, $\phi$ Lf and CTX $\phi(82,52$ and $40 \%$ similarity, respectively). It is noticeable that ORF411 has accumulated more mutations compared with its equivalent ORF384 of VGJ $\phi$ ( $76 \%$ similarity at the DNA level and $81.8 \%$ at the protein level). The significance of the evolutionary divergence of these ORFs is not known, but we speculate that it could be related to a better fitness of the phages for being hosted by different bacterial strains to evade mechanisms of resistance at the level of phage assembly. Immediately $3^{\prime}$ of ORF411 there is an intergenic region where major differences between VEJ $\phi$ and VGJ $\phi$ are present. In this region VGJ $\phi$ contains an insertion of $774 \mathrm{bp}$ with an ORF (ORF104) that is not present in VEJ $\phi$ (Fig. 1) and whose function is unknown. It seems that this is a more flexible
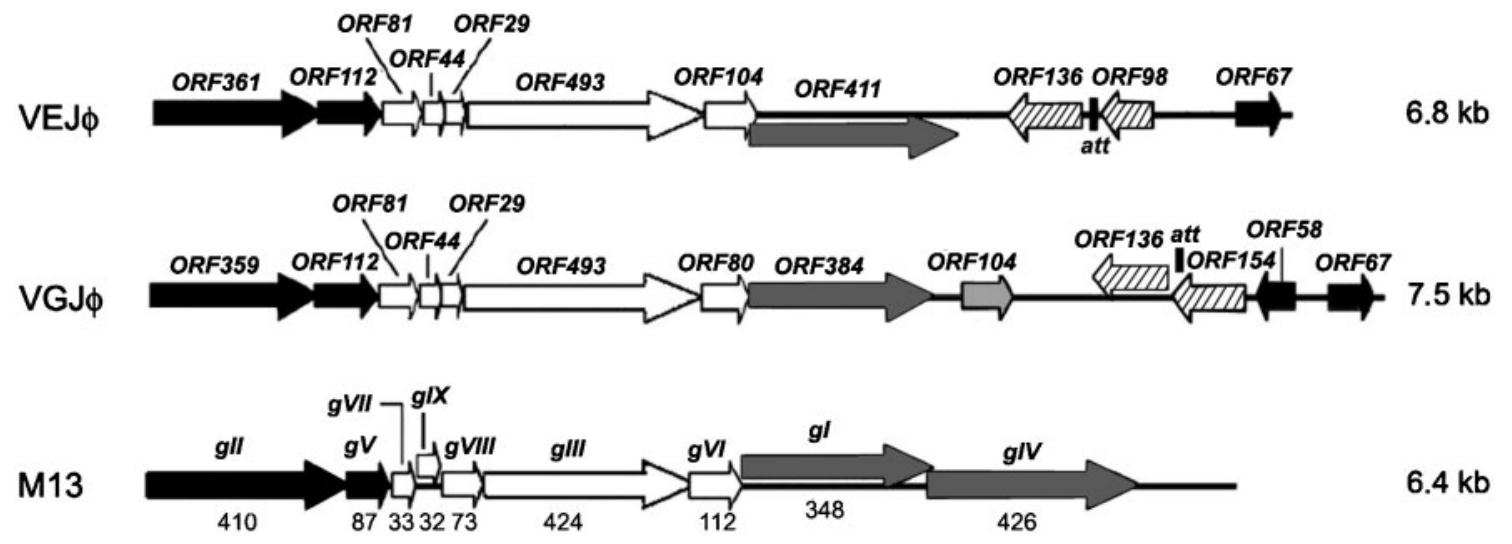

$6.4 \mathrm{~kb}$

Fig. 1. Modular structure of the genome of VEJ $\phi$ compared to those of phages VGJ $\phi$ of $V$. cholerae and M13 of E. coli. ORFs or genes are shown with arrows pointing in the direction of transcription. The number of amino acid residues encoded is shown in the name of each ORF, or below each gene in M13. The replication module is represented in black, the structural module in white, and the assembly and secretion module in dark grey. The ORFs belonging to the regulation module are hatched. The ORF104 of VGJ $\phi$, whose function is unknown, is represented in light grey. Genome sizes are shown on the right. 


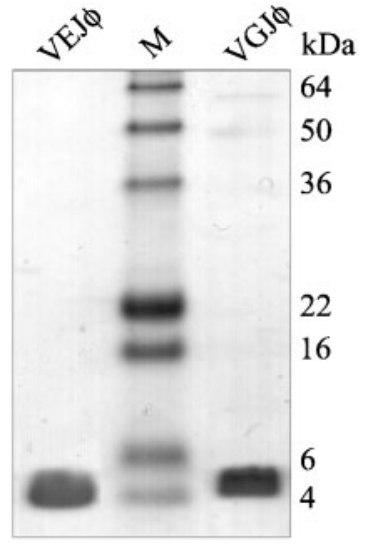

Fig. 2. Major capsid proteins of VEJ $\phi$ and VGJ $\phi$ visualized by SDS-PAGE. Purified viral particles of VEJ $\phi$ and VGJ $\phi$ were applied in the outer lanes, where the bands corresponding to the major capsid proteins are observed at $\sim 4 \mathrm{kDa}$. Protein molecular mass markers $(M)$ were applied in the middle lane and the size of each marker is shown to the right.

region of the genome, which can accommodate major changes without affecting the biological cycle of the phage and therefore favouring evolutionary fitness in the long term.

No gene homologous to $g I V$ of the Ff phage group of Escherichia coli, which encodes the secretin pIV needed for extrusion of the viral particles (Marciano et al., 1999), is present in VEJ $\phi$. Therefore, VEJ $\phi$ may rely upon a host protein for phage export, like CTX $\phi$ phage, which uses EpsD secretin of the type II secretion system (Davis et al., 2000).

The regulatory module of VEJ $\phi$ contains ORF136 and ORF98, which are encoded in the opposite direction with respect to the rest of the phage genes (Fig. 1). The product of ORF136 is $99.3 \%$ similar to the homonym hypothetical repressor of VGJ $\phi, 46.1 \%$ similar to repressor 1 of VSK and $35.1 \%$ to a lacI family repressor of Thermotoga maritima. The ORF98 product is $46.1 \%$ similar to repressor 2 of VSK and is almost identical to the aminoterminal region of the ORF154-encoded hypothetical repressor of VGJ申.

\section{VEJ $\phi$ uses the MSHA fimbria as a receptor}

Due to the similarity of VEJ $\phi$ with VGJ $\phi$, which uses the MSHA fimbria as a receptor, it was very probable that VEJ $\phi$ also uses this fimbria as a receptor. Therefore, we used the same procedure that was previously described to determine the VGJ $\phi$ receptor (Campos et al., 2003b). For this, we used the isogenic strains of $V$. cholerae KHT46 and KHT52 (Table 1) mutated at genes $m s h A$ or $t c p A$, coding for MSHA and TCP main pilin, respectively. Like VGJ $\phi$ phage, VEJ $\phi$ was able to infect only the strains with an intact MSHA fimbria and unable to infect the $m s h A$ mutant KHT46, clearly indicating that VEJ $\phi$ uses MSHA as a receptor.

\section{The integration site of VEJ $\phi$}

Some filamentous phages of $V$. cholerae are able to integrate into the bacterial genome (Campos et al., 2003b; Kar et al., 1996; Waldor \& Mekalanos, 1996). In the case of CTX $\phi$ it has been proven that integration is catalysed by recombinases XerCD (Val et al., 2005), which use as a substrate the genomic ssDNA of the phage for recombination with the bacterial chromosomal dif1 site (Val et al., 2005). VGJф is also able to integrate into the dif 1 site, as well as to form a cointegrate with the genome of CTX $\phi$ (Campos et al., 2003a, b). It is highly probable that XerCD also catalyse recombination reactions in these cases. Based on these antecedents we identified the sites similar to dif1 in VEJ $\phi$ and other integrative phages, which were possible substrates for XerCD catalysis. Taking the dif1 site of $V$. cholerae as a template, we found candidate sites on the negative strand of phages VEJ $\phi$, VGJ $\phi$ and VSK, which were found to be almost identical and which differed by only a few bases from the dif1 site (Fig. 3).

The putative XerC-binding sites differ in two bases from dif1, while the putative XerD-binding sites are identical in every phage and differ in two bases from difi. The central regions consist of seven nucleotides in place of six in dif1, and these are identical in the $5^{\prime}$ portion, where every one contains the TGT triplet (Fig. 3). However, the 3' extremes of the central regions differ significantly from difl. The $\mathrm{XerC}$ - and $\mathrm{XerD}$-binding sites, as well as the central region, are identical in VGJ $\phi$ and VEJ $\phi$; therefore VEJ $\phi$ is probably able to integrate into the bacterial chromosome as does VGJ $\phi$, although this has not been tested yet.

\begin{tabular}{|c|c|c|c|}
\hline & XerC & & XerD \\
\hline$V$. & AGTGCGCATTA & TGTATG- & TTATGTTAA \\
\hline - VEJ $\phi$ & ACTTCGCATTA & TGTCGGC & TTATGGTAA \\
\hline & ACTTCGCATTA & TGTCGGC & TTATGGTAA \\
\hline & ACTTCGCAGTA & TGTCGGC & TTATGGTAA \\
\hline
\end{tabular}

Fig. 3. Sequence alignment of the dif1 site of $V$. cholerae with similar sites encoded on the negative DNA strand of VEJ $\phi$, VGJ $\phi$ and VSK. Identical bases for each position are highlighted in grey. The probable binding sites for the recombinases XerC and XerD are shown. 


\section{VEJ $\phi$ transduces CTX $\phi$}

The finding of binding sites for XerCD recombinases in VEJ $\phi$ identical to those of VGJ $\phi$ and similar to the dif site of chromosome I of $V$. cholerae strongly suggested that VEJ $\phi$ could transduce CTX $\phi$ and hence the CT genes by forming a cointegrate between their genomes, as does VGJ $\phi$ (Campos et al., 2003a). To determine whether VEJ $\phi$ was able to transduce CTX $\phi$ we used a variant of CTX $\phi$ labelled with a kanamycin-resistant gene (CTX-Kn $\phi)$, so that transduction events could be followed by the transmission of the resistant phenotype between bacterial strains. V. cholerae strain 569B was infected with CTX-Kn $\phi$ or VEJ $\phi$, or co-infected with both phages. These three variants were used as donor strains of phage particles to infect strains KHT46 and KHT52 (isogenic mutants lacking MSHA and TCP pili, respectively), as well as 569B itself (Table 2). As expected, 569B infected with CTX-Kn $\phi$ transmitted the kanamycin-resistant trait to $569 \mathrm{~B}$, which expresses the CTX $\phi$ receptor TCP, but was unable to infect KHT52, the TCP-defective mutant (Table 2). Although KHT46 expresses TCP, this strain was not infected because it contains the CTX $\phi$ prophage of the El Tor group that confers phage immunity (Kimsey \& Waldor, 1998). However, 569B co-infected with CTX $\phi$ and VEJ $\phi$ was able to transfer the kanamycin-resistant trait to virgin 569B and to KHT52 (Table 2). Since KHT52 does not express TCP, and its isogenic MSHA-negative relative, KHT46, was unable to be infected by VEJ $\phi$, the transfer of the kanamycin trait without direct cell-cell contact likely proceeds through the MSHA fimbria by a phage containing the CTX-Kn $\phi$ genome or a part of it containing the $\mathrm{Kn}^{\mathrm{r}}$ gene. Donor strain 569B infected with VEJ $\phi$ alone, used as negative control, was unable to transfer the kanamycinresistant trait to any of the recipient strains (Table 2).

Due to the similarity between VEJ $\phi$ and VGJ $\phi$, we expected that VEJ $\phi$ uses the same mechanism of CTX $\phi$ transduction used by VGJ $\phi$ (Campos et al., 2003a), i.e. the formation of a hybrid phage genome between CTX $\phi$ and VEJ $\phi$ genomes. To study the mechanism of CTX $\phi$ transduction mediated by VEJ $\phi$, we made plasmid DNA preparations from 12 of the $\mathrm{Kn}^{\mathrm{r}}$ transductants of KHT52 from three independent experiments. The DNAs were digested with $\mathrm{NcoI}$, which cuts once into VEJ $\phi$ RF but does not cut into CTX-Kn $\phi$
RF; this produced two bands, of $\sim 15.0$ and $6.8 \mathrm{~kb}$, respectively (not shown). These results indicated the simultaneous presence of two plasmids: the VEJ $\phi$ RF of $6.8 \mathrm{~kb}$ and a larger plasmid, whose size $(15.0 \mathrm{~kb})$ was compatible with a cointegrate structure comprising both VEJ $\phi$ and CTX-Kn $\phi$ RFs fused into a single recombinant molecule, thus supporting our hypothesis. We designated the larger plasmid pHybP-VEJ/CTX $\phi$ (from hybrid phage). Electroporation of these plasmid DNA preparations (containing both plasmids) into KHT46 allowed us to propagate and purify clones containing $\mathrm{pHybP}-\mathrm{VEJ} / \mathrm{CTX} \phi$ alone under Kn selection (VEJ $\phi$ was washed out due to lack of its receptor, MSHA, in this strain). Finally, DNA sequencing of the novel junctions of pHybP-VEJ/CTX $\phi$ showed that they were produced by recombination of the XerCD sites of both phages' genomes (Fig. 4). This confirmed our hypothesis of the hybrid genome and showed that the genomes of both phages were oriented in opposite directions in the hybrid RF (Fig. 4).

\section{Conclusions}

Seven filamentous bacteriophages hosted by $V$. cholerae have been described so far: CTX $\phi$, fs1, fs2, VSK, 493, VGJ $\phi$ and KSF-1 (Campos et al., 2003b; Ehara et al., 1997; Faruque et al., 2005; Jouravleva et al., 1998; Kar et al., 1996; Nakasone et al., 1998; Waldor \& Mekalanos, 1996). Some of them have been implicated in the horizontal transfer of genetic material between strains of $V$. cholerae. The discovery that what was formerly called the CTX element is in fact the integrated genome of the filamentous phage CTX $\phi$ (Waldor \& Mekalanos, 1996) has profoundly modified the perspective with which scientists regard $V$. cholerae. It implies the existence of a horizontal transfer mechanism of the CT genes, which potentially pose the risk of virulence conversion of non-toxigenic environmental strains of $V$. cholerae. However, transduction of CT genes mediated by CTX $\phi$ depends on the expression of the phage receptor TCP by $V$. cholerae. An alternative mechanism of transmission of the CT genes, not dependent on TCP, is the one mediated by VGJ $\phi$, through which these genes could be acquired by non-toxigenic strains not sensitive to CTX $\phi$ infection (Campos et al., 2003a). This mechanism depends on the formation of a hybrid genome between

Table 2. Evaluation of CTX $\phi$ transduction to different strains mediated by VEJ $\phi$

\begin{tabular}{|c|c|c|c|}
\hline \multirow[t]{2}{*}{ Donor strain* } & \multicolumn{3}{|c|}{ Recipient strain $\dagger$} \\
\hline & KHT46 (MSHA $\left.{ }^{-}\right)$ & KHT52 $\left(\mathrm{TCP}^{-}\right)$ & $569 \mathrm{~B}$ \\
\hline 569B (CTX-Kn $\phi)$ & ND & ND & $4.1 \times 10^{6}$ \\
\hline 569B (CTX-Kn $\phi / \mathrm{VEJ} \phi)$ & ND & $5.5 \times 10^{4}$ & $4.2 \times 10^{5}$ \\
\hline 569B (VEJ $\phi)$ & ND & ND & ND \\
\hline
\end{tabular}

${ }^{\star}$ Cell-free culture supernatants of these strains were used for the infection assays.

$\nmid$ Recipient strains were grown under optimal conditions for the expression of the TCP receptor. The mean number of $\mathrm{Kn}^{\mathrm{r}}$ transductants of each recipient strain, from three independent assays, is shown. ND, Not detected. 


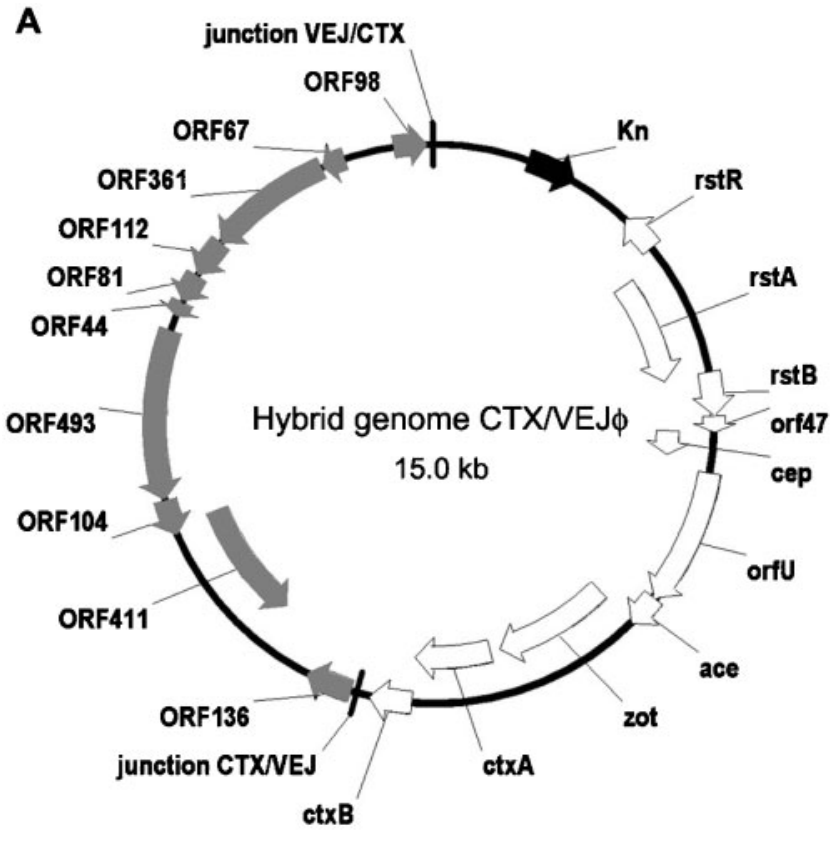

B

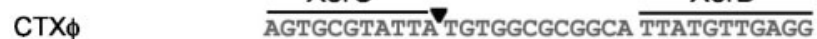

VEJ $\phi \quad$ ACTTCGCATTA TGTCG----GC- TTATGGTAAAA junction-CTXNEJ $\phi$ AGTGCGTATTA TGTCG----GC- TTATGGTAAAA junction-VEJ/CTX $\phi$ ACTTCGCATTA TGTGGCGCGGCA TTATGTTGAGG

Fig. 4. Structure of the hybrid genome formed by the recombination of VEJ $\phi$ with CTX-Kn $\phi$. (A) Map of the hybrid plasmid. Genes of CTX $\phi$ are shown in white, while genes of VEJ $\phi$ are in grey. The $\mathrm{Kn}^{r}$ gene is shown in black. The novel junctions at the boundaries of both genomes are also shown. (B) Nucleotide sequences of recombination sites of $\mathrm{CTX} \phi$ and $\mathrm{VEJ} \phi$ before site-specific recombination and sequences of the novel junctions after recombination, as determined by DNA sequencing. DNA sequences of CTX $\phi$ or VEJ $\phi$ origin are shown in grey or black, respectively. The XerC- and XerD-binding sites are indicated and the $\mathrm{XerC}$-cutting site is represented with an arrowhead.

those of VGJ $\phi$ and CTX $\phi$; thus a hybrid phage forms that uses MSHA as receptor (Campos et al., 2003a). MSHA is a fimbria more ubiquitously expressed among vibrios, increasing the risk of virulence conversion through this phage receptor.

In this work we characterized VEJ $\phi$, a new filamentous phage also capable of transmitting the CT genes among strains of $V$. cholerae expressing the MSHA receptor by the same mechanism as used by VGJ $\phi$. This constitutes another example of gene transfer dependent on phages in $V$. cholerae, in particular for the transmission of the CT genes, the main virulence factor of this pathogen. The greater variety of phages that use MSHA as a receptor compared with those that use TCP confirms that the former is a pilus much more accessible to phages in the environment. Other described phages, such as VSK, could also potentially transduce the CT genes, since we found that VSK contains integration sites similar to the dif site (Fig. 3). Thus, the diversity of phages capable of transducing genetic material in $V$. cholerae could be greater than formerly thought. The study of horizontal gene transfer in this bacterium is of great importance for understanding the future evolution of this pathogen and for the design of safer live attenuated vaccines.

\section{REFERENCES}

Campos, J., Martinez, E., Marrero, K., Silva, Y., Rodriguez, B. L., Suzarte, E., Ledon, T. \& Fando, R. (2003a). Novel type of specialized transduction for CTX $\phi$ or its satellite phage RS1 mediated by filamentous phage VGJ $\phi$ in Vibrio cholerae. J Bacteriol 185, 72317240.

Campos, J., Martinez, E., Suzarte, E., Rodriguez, B. L., Marrero, K., Silva, Y., Ledon, T., del Sol, R. \& Fando, R. (2003b). VGJ申, a novel filamentous phage of Vibrio cholerae, integrates into the same chromosomal site as CTXф. J Bacteriol 185, 5685-5696.

Davis, B. M. \& Waldor, M. K. (2003). Filamentous phages linked to virulence of Vibrio cholerae. Curr Opin Microbiol 6, 35-42.

Davis, B. M., Lawson, E. H., Sandkvist, M., Ali, A., Sozhamannan, S. \& Waldor, M. K. (2000). Convergence of the secretory pathways for cholera toxin and the filamentous phage, СTX $\phi$. Science 288, 333-335.

Ehara, M., Shimodori, S., Kojima, F., Ichinose, Y., Hirayama, T., Albert, M. J., Supawat, K., Honma, Y., Iwanaga, M. \& Amako, K. (1997). Characterization of filamentous phages of Vibrio cholerae O139 and O1. FEMS Microbiol Lett 154, 293-301.

Falero, A., Caballero, A., Ferrán, B., Izquierdo, Y., Fando, R. \& Campos, J. (2009). DNA binding proteins of the filamentous phages CTX $\phi$ and VGJ $\phi$ of Vibrio cholerae. J Bacteriol 191, 5873-5876.

Faruque, S. M., Asadulghani, Kamruzzaman, M., Nandi, R. K., Ghosh, A. N., Nair, G. B., Mekalanos, J. J. \& Sack, D. A. (2002). RS1 element of Vibrio cholerae can propagate horizontally as a filamentous phage exploiting the morphogenesis genes of CTX $\phi$. Infect Immun 70, $163-170$.

Faruque, S. M., Zhu, J., Asadulghani, Kamruzzaman, M. \& Mekalanos, J. J. (2003). Examination of diverse toxin-coregulated pilus-positive Vibrio cholerae strains fails to demonstrate evidence for Vibrio pathogenicity island phage. Infect Immun 71, 2993-2999.

Faruque, S. M., Bin Naser, I., Fujihara, K., Diraphat, P., Chowdhury, N., Kamruzzaman, M., Qadri, F., Yamasaki, S., Ghosh, A. N. \& Mekalanos, J. J. (2005). Genomic sequence and receptor for the Vibrio cholerae phage KSF-1申: evolutionary divergence among filamentous vibriophages mediating lateral gene transfer. J Bacteriol 187, 4095-4103.

Huber, K. E. \& Waldor, M. K. (2002). Filamentous phage integration requires the host recombinases XerC and XerD. Nature 417, 656-659.

Jouravleva, E. A., McDonald, G. A., Garon, C. F., BoesmanFinkelstein, M. \& Finkelstein, R. A. (1998). Characterization and possible functions of a new filamentous bacteriophage from Vibrio cholerae O139. Microbiology 144, 315-324.

Kar, S., Ghosh, R. K., Ghosh, A. N. \& Ghosh, A. (1996). Integration of the DNA of a novel filamentous bacteriophage VSK from Vibrio cholerae 0139 into the host chromosomal DNA. FEMS Microbiol Lett $145,17-22$.

Karaolis, D. K. \& Kaper, J. B. (1999). Vibrio cholerae TCP: a trifunctional virulence factor? Response. Trends Microbiol 7, 393.

Karaolis, D. K., Johnson, J. A., Bailey, C. C., Boedeker, E. C., Kaper, J. B. \& Reeves, P. R. (1998). A Vibrio cholerae pathogenicity island associated 
with epidemic and pandemic strains. Proc Natl Acad Sci U S A 95, 3134 3139.

Kiefer, D. \& Kuhn, A. (1999). Hydrophobic forces drive spontaneous membrane insertion of the bacteriophage Pf3 coat protein without topological control. EMBO J 18, 6299-6306.

Kiefer, D., Hu, X., Dalbey, R. \& Kuhn, A. (1997). Negatively charged amino acid residues play an active role in orienting the Secindependent Pf3 coat protein in the Escherichia coli inner membrane. EMBO J 16, 2197-2204.

Kimsey, H. H. \& Waldor, M. K. (1998). CTXф immunity: application in the development of cholera vaccines. Proc Natl Acad Sci U S A 95, 7035-7039.

Kostrikis, L. G., Reisberg, S. A., Kim, H. Y., Shin, S. \& Day, L. A. (1995). C2, and unusual filamentous bacterial virus: protein sequence and conformation, DNA size and conformation, and nucleotide/ subunit ratio. Biochemistry 34, 4077-4087.

Lee, C. A. (1999). Vibrio cholerae TCP: a trifunctional virulence factor? Trends Microbiol 7, 391-392.

Marciano, D. K., Russel, M. \& Simon, S. M. (1999). An aqueous channel for filamentous phage export. Science 284, 1516-1519.

Marvin, D. A. (1998). Filamentous phage structure, infection and assembly. Curr Opin Struct Biol 8, 150-158.

McMaster, G. K. \& Carmichael, G. G. (1977). Analysis of single- and double-stranded nucleic acids on polyacrylamide and agarose gels by using glyoxal and acridine orange. Proc Natl Acad Sci U S A 74, 48354838 .
Mendez, C. \& Chater, K. F. (1987). Cloning of whiG, a gene critical for sporulation of Streptomyces coelicolor A3(2). J Bacteriol 169, 5715-5720.

Nakasone, N., Honma, Y., Toma, C., Yamashiro, T. \& Iwanaga, M. (1998). Filamentous phage fs1 of Vibrio cholerae O139. Microbiol Immunol 42, 237-239.

Rajanna, C., Wang, J., Zhang, D., Xu, Z., Ali, A., Hou, Y. M. \& Karaolis, D. K. (2003). The vibrio pathogenicity island of epidemic Vibrio cholerae forms precise extrachromosomal circular excision products. J Bacteriol 185, 6893-6901.

Sambrook, J., Fritsch, E. F. \& Maniatis, T. (1989). Molecular Cloning: a Laboratory Manual. Cold Spring Harbor, NY: Cold Spring Harbor Laboratory.

Serek, J., Bauer-Manz, G., Struhalla, G., van den Berg, L., Kiefer, D., Dalbey, R. \& Kuhn, A. (2004). Escherichia coli YidC is a membrane insertase for Sec-independent proteins. EMBO J 23, 294-301.

Thelin, K. H. \& Taylor, R. K. (1996). Toxin-coregulated pilus, but not mannose-sensitive hemagglutinin, is required for colonization by Vibrio cholerae $\mathrm{O} 1 \mathrm{El}$ Tor biotype and $\mathrm{O} 139$ strains. Infect Immun 64, 2853-2856.

Val, M. E., Bouvier, M., Campos, J., Sherratt, D., Cornet, F., Mazel, D. \& Barre, F. X. (2005). The single-stranded genome of phage CTX is the form used for integration into the genome of Vibrio cholerae. Mol Cell 19, 559-566.

Waldor, M. K. \& Mekalanos, J. J. (1996). Lysogenic conversion by a filamentous phage encoding cholera toxin. Science 272, 1910-1914.

Edited by: V. Sperandio 